\title{
Capacidades y género: ¿Suma o sistema de desigualdades? El caso chileno
}

\author{
Virginia Guzmán, Emmanuelle Barozet, Eduardo Candia, \\ Bernardita Ibnen y Bettina Leiva
}

RESUMEN

Sobre la base del concepto amplio de capacidades propuesto por Amartya Sen y mediante los datos de la Encuesta Nacional de Estratificación Social (ENES) de 2009, en este artículo se explora la influencia del sexo en las diferencias de capacidades de los individuos consideradas importantes para lograr resultados que determinan sus niveles de bienestar o deprivación. En términos de capacidades, se investiga la habilidad de los individuos para tener control material, cultural, social y político de su vida y entorno, distinguiendo la situación de hombres y mujeres. Una vez construidas las medidas individuales de capacidades, se probó la hipótesis de la desventaja femenina a través del ajuste de modelos multivariados en los que se incluían controles relevantes. Los resultados obtenidos sugieren que en Chile existen diferencias no despreciables en capacidades entre mujeres y hombres.

PALABRAS CLAVE

CLASIFICACIÓN JEL

AUTORES
Desarrollo humano, investigación sobre el género, mujeres, desarrollo de capacidad, igualdad de género, medición, encuestas sociales, metodología estadística, Chile

D63, J16

Virginia Guzmán es investigadora del Centro de Estudios de la Mujer. virgguzman@gmail.com

Emmanuelle Barozet es profesora asociada del Departamento de Sociología de la Universidad de Chile. ebarozet@uchile.cl

Eduardo Candia es investigador asociado del Proyecto Desigualdades. ecandia@uc.cl

Bernardita Ihnen es estudiante de Sociología de la Universidad de Chile y practicante del Proyecto Desigualdades. berni.ihnen@gmail.com

Bettina Leiva es estudiante de Economía de la Universidad de Santiago de Chile y practicante del Proyecto Desigualdades. bettinaleivabianchi@gmail.com 


\section{I}

\section{Introducción}

Desde la década de 1970, numerosas investigaciones realizadas por centros de estudios, universidades y organismos internacionales, así como las efectuadas en Chile por organismos del Estado: Centro de Estudios de la Mujer (CEM), Servicio Nacional de la Mujer (SERNAM), Departamento de Estudios de la Dirección del Trabajo, y en América Latina (División de Asuntos de Género de la CePAL, Programa de las Naciones Unidas para el Desarrollo (PNUD)), han puesto de manifiesto la existencia de desigualdades ${ }^{1}$ entre hombres y mujeres en menoscabo de estas, identificando los factores que inciden en la desigualdad de género observables en distintos campos ${ }^{2}$. El propósito de este artículo es dar un paso más y analizar las consecuencias de las desigualdades de género en el desarrollo de capacidades y funcionamientos de los individuos, que condicionan la autonomía de las personas para generar elecciones concernientes a su bienestar individual y social.

Para ello, se partirá de los conceptos de capacidades y funcionamientos desarrollados por Amartya Sen (1985), que permiten preguntarse no solo por una hipotética

\footnotetext{
$\square$ Este artículo se enmarca en el Proyecto Anillos SOC12 inserto en el Proyecto Desigualdades (www.desigualdades.cl) y financiado por la Comisión Nacional de Investigación Científica y Tecnológica (CONICYT). Los autores agradecen los comentarios de Vicente Espinoza, Soledad Herrera, Javier Núñez y de un revisor de la Revista CEPAL. 1 "Una desigualdad social es el resultado de una distribución desigual, en el sentido matemático de la expresión, entre los miembros de una sociedad, de los recursos de ésta" (Bihr y Pfefferkorn, 2008, pág. 9). Obviamente, el término "desigualdad social" requiere de una importante precisión en cuanto al tipo de variables que se tomarán en cuenta para definir los distintos aspectos de dicha desigualdad. Se puede tratar cada una de estas dimensiones por separado o mediante una combinación de varias de ellas.

2 Para una exposición de estos temas existe una amplia literatura referida a los distintos ámbitos de desigualdad, como la participación económica (Contreras, Puentes y Bravo, 2005; Contreras y Plaza, 2010), la educación (Larrañaga, 2001; Contreras, 2004; Guerrero, Provoste y Valdés, 2006a y 2006b; MINEDUC, 2007), la segregación horizontal y vertical en el mercado de trabajo (Todaro y Yánez, 2004; Acosta, Perticará y Ramos, 2005), la calidad de empleo (Leiva, 2000; Acosta, Perticará y Ramos, 2005), el monto de los ingresos (Le Foulon y Beyer, 2002; Berstein y Tokman, 2005; Perticará y Bueno, 2009), las características de las trayectorias laborales (Guzmán y Mauro, 2004), el acceso a las pensiones (Bertranou y Arenas, 2003; Bertranou, 2005), la distribución del tiempo entre trabajo productivo y reproductivo (Todaro y Yánez, 2004; Dussaillant, 2009), la participación social (Osborne, 2005; Lombardo, 2008; PNUD, 2010), así como la participación y la representación política (Valdés, 2000; Altman, 2004; Ríos, 2008; PNUD, 2010).
}

repartición de bienes con equidad en una determinada sociedad, sino también avanzar en la comprensión de las diferencias que existen entre individuos a la hora de ejercer su libertad de ser y actuar, así como de tener acceso a los bienes disponibles. En tal sentido, este autor se aleja del universalismo abstracto del sujeto para estudiar a individuos concretos situados históricamente, en este caso, mujeres y hombres que acceden diferencialmente a los bienes según su posición dentro de las relaciones sociales en los distintos ámbitos de la sociedad. Los antecedentes proporcionados por los estudios de Nancy Fraser (1989 y 1990) y Fraser y otros (1994) sobre las dimensiones de la injusticia de género permiten igualmente superar una concepción que reduce el origen de las desigualdades a la distribución de los bienes, sin considerar otras dimensiones presentes en la generación de las desigualdades en cuanto al reconocimiento social vinculadas a las jerarquías de estatus y a la participación paritaria en las decisiones sociales y políticas.

El propósito entonces para el caso de Chile es construir cuatro capacidades — capacidad material, capacidad cultural, capacidad social y capacidad política- que serán tomadas como marco de referencia para comparar las medidas individuales de hombres y mujeres. Desde el punto de vista del tratamiento de los datos, se utilizaron modelos de regresión que permitieron estudiar las diferencias de capacidades entre hombres y mujeres, controlando por el efecto de variables sociodemográficas relevantes y otras capacidades. Para la medición de las capacidades en el caso de Chile, en el estudio se hace uso de datos primarios de la Encuesta Nacional de Estratificación Social (ENES) del Proyecto Anillos $\mathrm{SOC}_{12}{ }^{3}$. Esta encuesta presenta indudables ventajas

\footnotetext{
${ }^{3}$ Las reflexiones presentadas en este artículo forman parte de un campo de investigación mayor sobre igualdades y desigualdades en Chile, realizada por un equipo multidisciplinario y multiinstitucional cuya meta consiste en estudiar y medir, de manera comparada, las tendencias emergentes en la estratificación social chilena (www.desigualdades.cl). En este programa de estudio se examinan las variadas dimensiones de las desigualdades, no solo las derivadas de las dimensiones tradicionalmente reconocidas como la ocupación, el ingreso o el nivel educacional, sino también aquellas que tienen que ver con el reconocimiento social y la participación de las personas y colectividades en asuntos y decisiones colectivas. Asimismo, se plantea el carácter construido sociohistóricamente de las desigualdades que ponen a las personas y colectividades
} 
debido a su cobertura y representatividad nacional y regional, por ser aplicada individualmente a hombres y mujeres, pero a la vez tener una ficha del hogar al que pertenecen, y porque incluye variables emergentes en el estudio de la estratificación social relativas al capital social, cultural, cognitivo y político.

Dos son las hipótesis planteadas en este trabajo: i) en promedio las mujeres poseen menos capacidades en comparación con los hombres, aun controlando mediante predictores relevantes de las capacidades; ii) en la determinación de las capacidades, la variable sexo interactúa con otras variables explicativas que afectan

en posiciones de poder diferenciado en los distintos sistemas de relaciones sociales que estructuran la sociedad: de clase, de género, étnicas, espaciales, generacionales, entre otras.

\section{II}

\section{Antecedentes}

\section{Un sistema social de género}

En la teoría de género se afirma la existencia de un sistema social basado en la reproducción regular — con mayores o menores alteraciones- de patrones recurrentes que organizan las relaciones entre hombres y mujeres a nivel simbólico, normativo y práctico, en distintos espacios sociales e institucionales. El sistema de género se caracteriza por una gran extensión temporal y espacial, al comprender el conjunto de interacciones entre hombres y mujeres en la familia, el mercado, la política y la cultura, y por tener sus raíces en la constitución de las sociedades modernas (Valcárcel, 1991; Astelarra, 2003). Al abarcar la casi totalidad de los espacios sociales, y dado su amplio horizonte temporal, el sistema social de género tiene una gran capacidad de repercutir en otros sistemas sociales, y también la posibilidad de ser influido por ellos. Desde una perspectiva sociohistórica, el sistema social de género - como hoy se conoce- tiene sus raíces en los inicios de las sociedades modernas: se puede apreciar en la separación radical de los sexos en los discursos hegemónicos de la institución imaginaria de la modernidad, en la división sexual del trabajo, en las jerarquías de género, en la dicotomía privado-público, aunque no eliminan su influencia en las diferencias de capacidades encontradas entre hombres y mujeres.

Este artículo se estructura de la siguiente manera. En la sección II de antecedentes se revisa el enfoque de las capacidades tal como ha sido planteado por Sen, enfatizando el elemento multidimensional que implica la desigualdad y poniendo especial atención en los avances que han aportado los estudios sobre mujeres en el ámbito de las capacidades. En la sección III, se transita de la teoría a la medición en el ámbito de las capacidades. En la sección IV, se presentan los datos usados en la elaboración del modelo de análisis, así como la construcción de las cuatro capacidades que resumen gran cantidad de información respecto de la situación de las mujeres en Chile; asimismo, se discuten las relaciones con el marco sobre capacidades presentado previamente. En la sección V se ofrecen las conclusiones del análisis a la luz de las discusiones anteriores. así como en los criterios de inclusión y exclusión de la estructura de derechos que ordenan la ciudadanía.

En este contexto, en los desarrollos de Fraser (1989 y 1990) y Fraser y otros (1994) acerca de la "justicia de género" se distinguen distintas dimensiones relativamente autónomas entre sí: la dimensión económica de la redistribución, la dimensión cultural del reconocimiento y la dimensión política de la representación, las que integran en la noción más general de "justicia de paridad de participación". En el primer caso, las personas pueden verse impedidas de participar plenamente debido a las características de las estructuras económicas, que les niegan los recursos necesarios para interactuar con los demás como pares (injusticia distributiva). También pueden verse imposibilitadas de participar en condiciones de igualdad debido a la existencia de jerarquías institucionalizadas del valor cultural, que les rehúsan una posición adecuada, sufriendo en este caso una desigualdad de estatus o reconocimiento fallido. La tercera dimensión, el ámbito de lo político, remite a la jurisdicción del Estado y a las reglas que organizan la confrontación política. Lo político, en este sentido, suministra el escenario donde se desarrollan las luchas de las personas por la distribución y el reconocimiento. 


\section{El enfoque de las capacidades: de la justicia distributiva a la filosofía del desarrollo}

Varios son los enfoques desarrollados para explicar las desigualdades en la distribución de los bienes materiales y simbólicos en las sociedades. Entre los más característicos cabe mencionar a Rawls (1971), exponente de la concepción clásica de la justicia social que abstrae al individuo de su entorno, y a Kymlicka (1991), más cercano a Sen al considerar la influencia de los trasfondos culturales de las sociedades en la distribución de los bienes. A partir de 1980, Sen propone una aproximación conceptual y metodológica para abordar el tema del desarrollo y las desigualdades, y para ello analiza la singularidad y las diferencias radicales entre las personas a la hora de ejercer su libertad de ser y actuar, así como de tener acceso a los bienes disponibles en una sociedad.

Para entender cómo los individuos pueden ejercer su libertad, Sen distingue la capacidad (capability), por una parte, y el funcionamiento (functioning), por otra (Sen, 1977, 1979, 1985). La capacidad corresponde a la libertad que tiene el individuo para hacer o para ser, y para vivir una buena vida sobre la base de su decisión autónoma y sus propios valores: “...la capacidad es un tipo de libertad: la libertad fundamental para conseguir distintas combinaciones de funciones (o, en términos menos formales, la libertad para lograr diferentes estilos de vida)" (Sen, 2000, pág. 100). El concepto de capacidad se refiere a las "libertades sustanciales" con que una persona cuenta para desarrollar aquellas funciones que, según se piensa, tienen valor ${ }^{4}$. Los funcionamientos conciernen al grado en que las personas han podido desarrollar y ejercer las capacidades consideradas básicas para asegurar un desarrollo humano. Las capacidades y los funcionamientos juegan entonces un papel central para definir los niveles de bienestar y autonomía de los individuos.

En buenas cuentas, en la concepción de Sen se procura aprehender la singularidad de cada individuo como un yo situado, en sus circunstancias concretas, lo que implica enriquecer la noción de igualdad con la diversidad. Con ello se contrarrestan los riesgos de la estandarización derivada de la abstracción de la noción de sujeto o de una visión homogeneizadora o demasiado universalista del ser humano. De este modo, si bien para Sen dos sujetos pueden tener el mismo haz de bienes primarios, ellos pueden gozar de diferentes libertades

4 Véase un resumen de críticas al concepto y sus ampliaciones en Clark (2005). para perseguir sus respectivas concepciones de lo que es bueno. De igual manera, un mismo ingreso puede dejar subsistir mucha desigualdad respecto de la capacidad de llevar a cabo lo que se valora ${ }^{5}$.

\section{El enfoque de las capacidades aplicado al análisis de las desigualdades de género}

El género es uno de los campos donde la desigualdad es más difícil de evaluar, porque si bien existe abundante evidencia, tanto en los países desarrollados como en los en desarrollo, de que las mujeres están en peor situación que los hombres en una serie de dimensiones, no es fácil determinar cuál es el mejor indicador de dicha desigualdad. En el enfoque de Sen no todas las desigualdades son negativas, pero sí algunas de sus formas son perjudiciales: "si aquellos elementos que no se derivan del esfuerzo personal (género, raza, procedencia del hogar) son los que explican la desigualdad, entonces la desigualdad no es aceptable. Pero si la desigualdad es producto del esfuerzo de las personas, controlando las otras dimensiones, entonces esa desigualdad es aceptable" (Gamero Requena, 2006, pág. 89). En esta ecuación, el género es una de las variables que más parece influir en el desigual acceso a recursos y al ejercicio de la libertad, formando parte de las desigualdades no aceptables. En este contexto, una gran cantidad de autores retomaron el desafío planteado por Sen (Anand, Hunter y Smith, 2005) e intentaron elaborar, de manera más o menos fija, más o menos local o universalista, una lista de capacidades que respondan a la realidad de hombres y mujeres. Existe en este ámbito un extenso debate respecto de la validez del hecho de establecer una lista, puesto que el mismo Sen ha abogado por la necesidad de ser flexible y de definir dicha lista siempre en un contexto, un lugar y un espacio determinados, evitando así listas más bien teóricas o a priori. De hecho, esta misma flexibilidad que muestra el aporte de Sen, así como su grado de pluralismo interno, es sin lugar a dudas uno de los elementos que permiten a los investigadores realizar aplicaciones fructíferas de esa teoría.

\footnotetext{
5 Por ejemplo, no todos los individuos — aun teniendo los mismos recursos y capacidades - elegirán aplicar sus capacidades de la misma manera y por lo tanto no tendrá los mismos funcionamientos. El entorno y las limitaciones que este impone, además de las decisiones del propio individuo — su capacidad de agencia, es decir, en lenguaje de Sen, la capacidad "de la persona que actúa y provoca cambios y cuyos logros pueden juzgarse en función de sus propios valores y objetivos" (Sen, 2000, pág. 35)— también introducen importantes variaciones en el ejercicio de la libertad.
} 
Sobre esta base, Nussbaum (1995, 2000, 2003 y 2005) y Robeyns (2003 y 2006) recurren a la teoría de Sen para analizar las diferencias de capacidades entre hombres y mujeres, profundizando de esta manera en los muchos esfuerzos que se han hecho para completar, hacer operativa o especificar la lista de capacidades. Atendiendo a la producción de la teoría feminista, estas autoras reconocen que las jerarquías de género, la dicotomía privado-público y los criterios de inclusión y exclusión de la estructura de derechos que conforman la ciudadanía han derivado en que las mujeres estén poco presentes en la participación pública, en los sistemas de representación política y en la titularidad de los derechos. Confinadas sobre todo al mundo doméstico, donde también se ubican las prácticas de reproducción biológica y social, sus experiencias y problemas no son habitualmente considerados materias sobre las que se puedan tomar decisiones colectivas y deben ser mediadas por la posición de sus parejas (Goldthorpe, 1983). De esta forma, la responsabilidad principal respecto de las tareas de cuidado las somete permanente a las decisiones y necesidades de los otros, mermando su libertad. Un orden social estructurado en torno de esta división sexual del trabajo y de representaciones de lo femenino y masculino resta oportunidades a las mujeres para desarrollar las capacidades, lo que se refleja en los distintos funcionamientos de capacidades de hombres y mujeres.
Uno de los esfuerzos más acabados por fijar la lista de capacidades de manera universal ha sido el de Nussbaum, que ha establecido una lista de 10 componentes separados que se relacionan íntimamente y que debieran estar asegurados para que una persona ejerza su libertad y acceda al bienestar: i) vida; ii) salud física; iii) integridad física; iv) sentidos, imaginación y pensamientos; v) emociones; vi) razón práctica; vii) asociación; viii) preocupación por otras especies; ix) recreación; y $\mathrm{x})$ control político y material sobre el ambiente propio (Nussbaum, 1995, 2000, 2003 y 2005). En el marco de la misma discusión, Robeyns — también a partir de la teoría de Sen- propone ocho componentes para estudiar las capacidades: i) vida y salud física; ii) bienestar mental, integridad y seguridad física; iii) relaciones sociales; iv) empoderamiento político; v) educación y conocimiento; vi) trabajo doméstico y cuidado no mercantil; vii) abrigo y alojamiento y medio ambiente; y viii) movilidad (Robeyns, 2003 y 2006). Los aportes de ambas autoras, considerados a la fecha como los más avanzados en este campo poseen, sin embargo, una diferencia fundamental: Nussbaum — fiel a la tradición de la filosofía moral— procuró establecer una lista que pudiera ser válida en más de un contexto, mientras que Robeyns ha intentado sistemáticamente aplicar la lista de acuerdo con las características de países concretos, sin pretender "exportarla" a otras situaciones.

\section{III}

\section{Capacidades y funcionamientos: de la teoría a la medición}

Mucho se ha escrito acerca de la dificultad no solo de definir las capacidades según el tipo de análisis que se quiere realizar, sino especialmente sobre cómo medirlas. La pregunta de la medición empírica de las capacidades ha dado lugar desde los años ochenta a un amplio debate (Anand, Hunter y Smith, 2005), sintetizado por las Naciones Unidas a través del índice de desarrollo humano (IDH). Dicha medición ha sido aplicada a diversos países a través de adaptaciones e innovaciones en las mediciones (Martinetti, 2000). Uno de los puntos centrales del debate tiene que ver con los datos disponibles, siendo muchas veces datos secundarios y por lo tanto algo lejanos al enfoque inicial (Anand, Hunter y Smith, 2005; Robeyns, 2006). Además, la mayor parte de las aplicaciones empíricas del enfoque de capacidades se ha realizado en países del norte, en especial los Estados Unidos de América y Europa (Alkire, 2002; Burchardt y Zaidi, 2003; Van Hees, 2004; Kuklys, 2005; Layte, Nolan y Whelan, 2000; Qizilbash, 1996; Schokkaert y Van Ootegem, 1990, entre otros). Existen escasas aplicaciones empíricas o medición de las capacidades en los países del sur (Clark y Qizilbash, 2005, sobre Sudáfrica), o en las potencias emergentes (Majumdar y Subramanian, 2001 para la India).

Una de las mayores dificultades en el trabajo empírico, compartida también por este estudio, es distinguir capacidades y funcionamientos, pues con datos reales esta distinción teórica se vuelve más tenue (Anand, 
Hunter y Smith, 2005). En muchos datos usados en este campo, y también en los datos que se usan en este trabajo, tanto las capacidades como los funcionamientos fueron abordados a través de las preguntas, ya sea sobre lo que las personas hacen (por ejemplo, si votan o no votan, su nivel educacional) o respecto de sus posibilidades de hacer (estudiar más, viajar, entre otras).

A partir del concepto de capacidad de Sen, de sus adaptaciones a la teoría de género y del análisis de las cuatro dimensiones de la injusticia de género de Fraser, en este trabajo se elaboran capacidades que fuesen suficientemente sensibles para captar los factores que generan desigualdad entre hombres y mujeres y que afectan a las libertades de las mujeres para desarrollar sus capacidades y alcanzar distintos perfeccionamientos. Se definen entonces cuatro categorías de capacidades para comparar a hombres y mujeres individualmente: capacidad material, capacidad social, capacidad cultural y capacidad política.

El concepto de capacidad material se refiere aquí a la habilidad individual de proveerse recursos en forma autónoma y de abordar, a través de sistemas de seguridad social, situaciones de riesgo y vulnerabilidad material originadas por el envejecimiento y problemas de salud.
El concepto de capacidad social hace mención a recursos sociales que una persona puede movilizar para incrementar su bienestar (por ejemplo, acceder a recursos materiales y simbólicos) o enfrentar eventualidades negativas.

El concepto de capacidad cultural alude a los recursos de los que disponen los sujetos para comprender su entorno y reflexionar en forma crítica sobre su vida y la sociedad.

Finalmente, la capacidad política hace referencia a la habilidad de las personas para participar e incidir en las decisiones políticas que competen a su vida y a la comunidad global.

Las capacidades así definidas otorgan a las personas recursos para consolidar su independencia material, desarrollar un conocimiento crítico de la realidad, confiar y relacionarse socialmente y, por último, participar de decisiones colectivas a nivel político e institucional. Por ende, ellas condicionan sus grados de autonomía y de seguridad para tomar decisiones relativas a su bienestar individual y colectivo, así como para anticipar situaciones de vulnerabilidad futura en cuatro grandes campos de la vida.

\section{IV}

\section{Datos, construcción de herramientas y métodos}

\section{Encuesta Nacional de Estratificación Social (ENES), 2009}

En este trabajo se utilizan datos de la Encuesta Nacional de Estratificación Social (ENES) del Proyecto Desigualdades para medir la movilidad y estratificación social a través de variables clásicas y otras no disponibles en instrumentos normalmente usados en el estudio de la estratificación social en Chile, como son las encuestas de hogares (Encuesta de Caracterización Socioeconómica Nacional (CASEN)). La ENES fue aplicada entre mayo y agosto de $2009^{6}$ a una muestra de 6.153 individuos (3.141 mujeres), pertenecientes a 3.365 hogares. La muestra es representativa de la población chilena de 18 años y más ${ }^{7}$, pues

\footnotetext{
${ }^{6}$ Encuesta realizada en terreno por la empresa Statcom Estadísticos Consultores Ltda.

7 Universo que corresponde a 11.965 .900 personas a junio de 2008, según proyecciones de población del Instituto Nacional de Estadísticas (INE).
}

corresponde a un diseño aleatorio, estratificado - por región y zona ${ }^{8}$ - por conglomerados en tres etapas, a saber, selección de manzanas, hogares e individuos, y con un error estimado para individuos a nivel nacional de 1,3\%, considerando varianza máxima y a un nivel de confianza del 95\%. En cada hogar se obtuvo información sociodemográfica general y para cada uno de sus miembros. Se encuestó hasta tres integrantes del hogar mayores de 18 años, incluido el principal proveedor de ingresos, su pareja — si aplicaba- y otro(s) sujeto(s) elegible(s) seleccionado(s) al azar. La aplicación se hizo en forma presencial por un encuestador profesional. El instrumento comprende 12 módulos que recogen información sobre identificación social y territorial, religión y viajes, trabajo, patrimonio, educación, movilidad social, capital social, posición y participación

\footnotetext{
${ }^{8}$ Corresponde a 45 estratos: 15 regiones y 3 zonas (centros urbanos, restos urbanos, rural). En el diseño se excluye a Isla de Pascua y Juan Fernández.
} 
política, valores, y situación personal y familiar. Cabe mencionar que en este trabajo se utilizó un subconjunto de 5.443 observaciones de las ENES, porque individuos que se declaraban como jubilados o discapacitados no eran elegibles para el análisis.

\section{Conceptos y mediciones}

Sobre la base de los antecedentes empíricos y teóricos presentados y a partir de una selección de variables incluidas en las ENES, se procedió a la construcción de índices que midieran cuatro capacidades específicas: capacidad material, capacidad cultural, capacidad social y capacidad política. En estos índices se combina una gran cantidad de información a nivel individual, incluidas condiciones, prácticas o conductas y actitudes, permitiendo así reducir la dimensionalidad del problema y la construcción de modelos de mayor parsimonia. A continuación se recuerda brevemente su definición conceptual y se presentan detalles de su construcción.

Retomando el concepto de "capacidad material", conviene recordar que este se refiere a la habilidad individual ${ }^{10}$ de proveerse recursos en forma autónoma y de abordar situaciones de riesgo y vulnerabilidad material originadas por el envejecimiento y problemas de salud, a través de sistemas de seguridad social. Se trata de un concepto relevante para la población en edad de trabajar que no se declara retirada, lo que corresponde a 5.443 observaciones de la muestra y al subconjunto de observaciones que se utilizarán en los análisis que siguen. La capacidad material está medida por un índice ordinal de tres categorías que distingue entre individuos con capacidad material baja (32\%), que no cuentan con empleo ni poseen activos ${ }^{11}$; capacidad material media (29\%), que tienen un empleo o un activo, pero sin seguridad social ${ }^{12}$; y capacidad material alta (39\%), que poseen un empleo o activos y simultáneamente seguridad

\footnotetext{
${ }^{9}$ La capacidad material, para remitir a un punto tratado anteriormente, es la única medida que no es de logro, sino que se trata de un resultado. ${ }^{10}$ No se trata de negar que existen personas que debido a la situación de su hogar logran un capital económico importante, más allá de sus capacidades individuales, como puede ocurrir en el caso de una dueña de casa casada con alguien de altos ingresos, lo que remite a la posibilidad de obtener una gran capacidad material sin trabajo formal ni activos por la vía de emparejamiento, a través de los hijos o por la familia extendida. Sin embargo, la elección de trabajar sobre capacidades individuales remite a lo que se obtiene de manera autónoma sin transitividad respecto de otras personas y que por lo tanto resiste a los cambios de situación familiar o cercanía con otras personas.

11 Tales como ser socio de un negocio, propiedades para arriendo, ganado, maquinaria, y otros.

12 Tener seguridad social corresponde aquí a cotizar en un sistema de previsión y tener acceso a un sistema de salud.
}

social. Cabe destacar que esta medida es independiente del nivel de ingresos o nivel de vida del hogar. De esta forma, dos personas que tienen montos de ingresos diferentes pueden entrar en una misma categoría de capacidad material, tal como se verá más adelante. La ventaja de su uso en el contexto de este estudio es que permite describir la autonomía material de las mujeres, independientemente de su participación laboral y del nivel de vida del hogar donde están insertas, ayudando así a resolver uno de los problemas de medición de la desigualdad en el caso de las mujeres.

A su vez, el concepto de "capacidad cultural" alude a los recursos de que disponen los sujetos para comprender su entorno y reflexionar en forma crítica sobre su vida. Está medido por un índice en que se combinan, por una parte, las dimensiones de años de escolaridad y dominio de un idioma extranjero (bajo, medio y alto), con una ponderación de 0,3 , respectivamente, en la escala total, y por otra, la frecuencia de viajes al extranjero y número de cursos realizados fuera de la educación formal o en el marco de capacitaciones, dimensiones con una ponderación de 0,2 cada una.

El concepto de "capacidad social" hace referencia a recursos sociales que una persona puede movilizar para incrementar su bienestar (por ejemplo, acceder a recursos materiales y simbólicos) o para enfrentar eventualidades negativas, tal como ha mostrado la teoría de redes (Granovetter, 1973; Porras y Espinoza, 2005). Está medido por un índice en que se combinan: diversidad de la red personal, dada por el número de conocidos que reporta un individuo de un conjunto de 12 ocupaciones diversas; participación asociativa, dada por la participación activa en al menos una asociación; y dos actitudes: compromiso comunitario y confianza interpersonal, que corresponden a variables latentes o no directamente observables y que en consecuencia requirieron un tratamiento especial ${ }^{13}$.

${ }^{13}$ Las medidas de estas actitudes se establecieron a partir de escalas definidas por medio de análisis factorial exploratorio (AF), es decir, con grupos de ítems que se asignaban claramente en un factor teóricamente interpretable. En este modelo se usó un conjunto de 10 ítems que indagaban sobre frecuencia de prácticas en actividades en beneficio de la comunidad y grado de confianza en distintas personas, todos con escalas de respuesta cuasimétricas. Para obtener este modelo de medición se usó AF con método de extracción de componentes principales y con rotación oblicua (oblimn), bajo el supuesto teórico de que los factores resultantes deberían estar correlacionados. Además, para minimizar la pérdida muestral según lista, se utilizó imputación de la media en ítems con información perdida. Se obtuvo una solución de dos factores interpretables, respectivamente, como compromiso comunitario y confianza. $\mathrm{La}$ escala de compromiso comunitario agrupa a seis ítems con pesos factoriales entre 0,60 y 0,75 y presenta un alfa de Cronbach de 0,75 
Finalmente, en el concepto de "capacidad política" se considera la habilidad de las personas para participar e incidir en las decisiones políticas que competen a su vida. Está medido por un índice en que se combinan: presencia de inscripción electoral declarada por el entrevistado; nivel de información política, dada por número de respuestas correctas a preguntas básicas de conocimientos sobre política ${ }^{14}$; y las actitudes: confianza en instituciones políticas, tendencia a conversar e informarse sobre política y propensión a participar en actividades políticas, cuyas medidas se construyeron con el mismo método usado con respecto a las variables latentes del índice de capacidad social.

En el cuadro 1 se muestran los estadísticos descriptivos ${ }^{15}$ para las medidas de capacidad construidas.

\section{Modelos y variables de control}

Para conocer el efecto del sexo en las medidas de capacidades construidas se utilizaron técnicas de análisis apropiadas a sus niveles de medición, ajustando modelos sucesivos que tomaban cada una de las capacidades como variables dependientes.

A fin de estudiar la relación del sexo con la capacidad material se usó un modelo logit para categorías

lo que indica un adecuado nivel de confiabilidad o consistencia interna de la escala. En la escala de confianza interpersonal se agruparon cuatro ítems con pesos factoriales entre 0,67 y 0,85 y un alfa de Cronbach de 0,79. Luego, mediante el método de regresión, se obtuvieron puntuaciones factoriales que asignan una medida resumen de cada actitud a cada observación en una escala continua. Para la construcción final de la medida de capacidad social, se combinaron sus cuatro dimensiones con igual ponderación (es decir, 0,25 cada una) en un índice que oscila teóricamente entre 0 y 100 . Este índice presenta una media de 29 puntos con una desviación estándar de 17 , para 5.443 observaciones válidas.

14 Específicamente: identificar correctamente el mecanismo de elección del gobierno regional e intendentes, e identificar el nombre de autoridades (alcalde, intendente y ministro del interior).

15 En todos los cálculos se utiliza una ponderación muestral (factor de expansión normalizado) que asegura que los estadísticos sean representativos del universo estudiado. múltiples, que en este caso estima la relación entre probabilidad de respuesta de una variable dependiente ordinal y un conjunto de variables explicativas categóricas y continuas. La variable de respuesta es el logaritmo de las oportunidades (odds) de una alternativa en relación con una categoría de referencia. Tomando el exponente de los parámetros aditivos de los predictores del modelo se obtienen parámetros multiplicativos, que pueden ser interpretados como la media geométrica del aumento $\left(\beta_{\mathrm{i}}>1\right)$ o disminución $\left(\beta_{\mathrm{i}}<1\right)$ de los odds de una categoría de la variable dependiente en relación con la categoría de referencia (por ejemplo, odds de capacidad material baja en comparación con la media), controlando por todos los otros factores del modelo. Los fuerza de los efectos está dada por la medida en que estos parámetros se alejan del valor 1 (siendo 0 el límite inferior), mientras que los parámetros cercanos a 1 indican ausencia de efecto (Billiet, 1995). Dado que la variable de respuesta tiene tres categorías por cada predictor continuo, se estiman dos parámetros, y por cada predictor categórico dos veces el número de categorías menos 1 .

La relación entre sexo y capacidad cultural, social y política se estudió con tres modelos respectivamente. La técnica usada fue la regresión lineal (mínimos cuadrados ordinarios). En este tipo de modelo, los parámetros ( $\beta$ en cuadros 6,7 y 8 a continuación) se interpretan como el cambio en el resultado (capacidad en este caso) relacionado con un cambio de una unidad en un predictor, controlando por todos los otros factores del modelo. Cuando un predictor es categórico, su parámetro se interpreta como cambio en el resultado asociado a una categoría con contraste con una categoría de referencia. Los $\beta$ estandarizados permiten comparar el tamaño del efecto entre variables independientes. En este caso, se probaron posibles interacciones entre sexo y otros predictores mediante un método de eliminación reversa de efectos.

Junto con el sexo, incluido como la variable muda mujer $(51,4 \%)$, sobre la base de consideraciones teóricas, en los modelos ajustados (tanto logit como regresión)

\begin{tabular}{lccccc}
\hline & Número de observaciones & Mínimo & Máximo & Media & Desviación típica \\
\hline Capacidad cultural & 5428 & 0 & 79,43 & 21,08 & 13,54 \\
Capacidad política & 5443 & 2,31 & 94,5 & 32,75 & 14,98 \\
Capacidad social & 5209 & 0,65 & 97,23 & 28,7 & 16,85 \\
\hline
\end{tabular}

Fuente: Encuesta Nacional de Estratificación Social (ENES), 2009. 
se incluyeron como variables independientes: edad en años cumplidos; hijos $<18$ años ${ }^{16}$, que indica si el entrevistado tiene hijos menores de edad (51,1\%); área rural, que señala si la residencia es zona rural $(13,1 \%)$; nivel socioeconómico, que corresponde al puntaje del hogar basado en disponibilidad de bienes y educación

${ }^{16} \mathrm{El}$ hecho de que una persona tenga hijos menores de 18 años es importante, pues influye en las capacidades y autonomía más bien de las mujeres, siendo esta una de las hipótesis fundamentales de los trabajos en este campo de estudio. del principal sostenedor ${ }^{17}$, indicativo del nivel de vida. Además, para la explicación de cada capacidad específica se incluyeron como predictores el resto de las capacidades. Esto permitió determinar el efecto neto del sexo en cada capacidad, controlando por un conjunto de variables sociodemográficas y otras capacidades asumidas como relevantes. En el cuadro 2 se resumen las estadísticas de estas variables.

${ }^{17}$ Según matriz de la Asociación Internacional de Marketing (AIM).

CUADRO 2

\section{Estadísticos resumen de las variables usadas}

\begin{tabular}{|c|c|c|c|c|c|}
\hline & Número de observaciones & Media & Desviación típica & Mínimo & Máximo \\
\hline Mujer & 5443 & 0,51 & 0,50 & 0 & 1 \\
\hline P84 ¿Cuántos años cumplidos tiene? & 5443 & 39,51 & 14,45 & 18 & 93 \\
\hline Tiene hijos $<18$ años & 5443 & 0,51 & 0,50 & 0 & 1 \\
\hline NSE08 (puntaje) & 5230 & 354,02 & 243,76 & 0 & 1000 \\
\hline Rural & 5443 & 0,13 & 0,34 & 0 & 1 \\
\hline
\end{tabular}

Fuente: Encuesta Nacional de Estratificación Social (ENES), 2009.

\section{V}

\section{Resultados}

\section{Diferencias observadas}

Un examen simple de las diferencias observadas por sexo en las medidas de capacidades construidas sugiere que las mujeres se encuentran en una situación de desventaja en cada una de estas. Como punto de partida, las mujeres muestran una capacidad material significativamente

CUADRO 3

Capacidad material en función del género (En porcentajes)

\begin{tabular}{lccc}
\hline $\begin{array}{l}\text { Capacidad } \\
\text { material }\end{array}$ & Hombres & Mujeres & $\begin{array}{c}\text { Número de } \\
\text { observaciones }\end{array}$ \\
\hline Baja & 18,3 & 45,3 & 1750 \\
Media & 28,9 & 28,2 & 1552 \\
Alta & 52,8 & 26,5 & 2137 \\
Total & 100,0 & 100,0 & 5439 \\
\hline
\end{tabular}

Fuente: Encuesta Nacional de Estratificación Social (ENES), 2009.

$\chi^{2}=545,52 ; \mathrm{df}=2,{ }^{*} \mathrm{p}<0,000 ;$ Gamma $=-0,49$ menor que los hombres. En este caso, el tamaño del efecto del sexo medido por el estadístico Gamma puede considerarse grande, como se indica en el cuadro 3.

En segundo lugar, como se indica a continuación, en promedio se observa en las mujeres una capacidad cultural, social y política significativamente menor que en los hombres; sin embargo, el tamaño del efecto es pequeño.

Enseguida se analizará en qué medida estas diferencias observadas se sostienen al modelar la relación sexo-capacidades, controlando por una serie de factores asumidos como relevantes. Cabe mencionar que en el ajuste de estos modelos (logit y regresiones lineares), el cálculo de errores típicos asociados a los parámetros de los predictores consideró el diseño muestral estratificado y por conglomerados utilizado en la selección de participantes de ENES.

\section{Efectos netos}

En el cuadro 5 se presenta el modelo para capacidad material. 
Diferencia de medias entre hombres y mujeres en capacidad cultural, social y políticaa

\begin{tabular}{|c|c|c|c|c|c|c|}
\hline & Media hombre & Media mujer & Estadístico t & $\begin{array}{l}\text { Grados de } \\
\text { libertad (gl) }\end{array}$ & $\begin{array}{l}\text { Significancia } \\
\text { (bilateral) }\end{array}$ & $\begin{array}{c}\text { Coeficiente de correlación } \\
\text { de Pearson } r\end{array}$ \\
\hline Capacidad cultural ${ }^{b}$ & 23,9 & 20,4 & 9,32 & 4814,9 & 0,00 & 0,13 \\
\hline Capacidad social ${ }^{b}$ & 30,4 & 27,1 & 7,45 & 5371,2 & 0,00 & 0,10 \\
\hline Capacidad políticab & 33,8 & 31,7 & 5,10 & 5338,0 & 0,00 & 0,07 \\
\hline
\end{tabular}

Fuente: Encuesta Nacional de Estratificación Social 2009.

a No se asume igualdad de varianza.

b No cumple con el supuesto de normalidad. La prueba no paramétrica arrojó resultados similares.

CUADRO 5

Modelo para capacidad material

(Parámetros multiplicativos)

\begin{tabular}{|c|c|c|c|}
\hline Predictores & $\begin{array}{c}\text { Baja comparada con } \\
\text { capacidad media }\end{array}$ & $\begin{array}{l}\text { Alta comparada con } \\
\text { capacidad media }\end{array}$ & $\begin{array}{c}\text { Chi-cuadrado } \\
\text { (grados de libertad) }\end{array}$ \\
\hline (Constante) & 0,710 & $2,347 * *$ & $72,15 * *(2)$ \\
\hline Mujer (Referencia: hombre) & $2,851 * * *$ & $0,524 * * *$ & $510,82 * *(2)$ \\
\hline Edad en años & $0,986 * *$ & 0,991 & $21,24 * *(2)$ \\
\hline Tiene hijos $<18$ años (Referencia: no tiene) & $0,545 * * *$ & 1,260 & $124,04 * *(2)$ \\
\hline Nivel socioeconómico & 1,000 & $1,001 * * *$ & $46,23 * *(2)$ \\
\hline Área rural (Referencia: área urbana) & 1,034 & 0,682 & $15,60 * *(2)$ \\
\hline Capacidad cultural & 0,988 & $1,012 *$ & $36,65 * *(2)$ \\
\hline Capacidad social & $0,985 * *$ & $0,990 * *$ & $38,17 * *(2)$ \\
\hline Capacidad política & 0,996 & $1,010 * *$ & $20,87 * *(2)$ \\
\hline
\end{tabular}

Fuente: Encuesta Nacional de Estratificación Social (ENES), 2009.

Pseudo $\mathrm{R}^{2}$ Cox y Snell = 0,188, pseudo $\mathrm{R}^{2}$ Nagelkerke $=0,212 ; * * * \mathrm{p}<0,001, * * \mathrm{p}<0,01, * \mathrm{p}<0,05$.

Estos resultados permiten ver que aun luego de controlar por el efecto del resto de las variables, la variable mujer presenta la correlación más importante. El efecto es significativo, funciona en la dirección esperada (esto es, desventaja para las mujeres) y puede considerarse moderado. De acuerdo con los parámetros, en comparación con un hombre, una mujer tiene en promedio cerca de tres veces más oportunidades de estar en la categoría de capacidad material baja comparada con media, y dos veces menos oportunidades de pertenecer a la categoría de capacidad material alta comparada con la media. Por otra parte, tener hijos menores de edad resulta la segunda variable en importancia en el modelo. Esta condición casi duplica $(1 / 0,55)$ las posibilidades de tener capacidad material media versus baja, mientras que habitar el área rural reduce en 1,5 veces $(1 / 0,68)$ las oportunidades de capacidad alta en comparación con la media. El resto de los predictores, particularmente nivel socioeconómico y las otras capacidades, presentan relaciones débiles con capacidad material, es decir, con parámetros muy cercanos a 1 , pese a resultar en ocasiones estadísticamente significativas ${ }^{18}$.

En el cuadro 6 se presenta el modelo de capacidad cultural.

\footnotetext{
${ }^{18}$ Esto sucede cuando se obtiene un valor alto en el estadístico Wald usado para testear la contribución de cada predictor. Dicho estadístico se calcula como el cuadrado de la razón entre el parámetro aditivo de cada predictor y su error estándar y asumiendo que sigue una distribución de chi-cuadrado (Garson, 2011).
} 
CUADRO 6

Modelo para capacidad cultural

\begin{tabular}{|c|c|c|c|}
\hline Predictores & $\beta$ & Error típico linealizado & $\beta$ estandarizado \\
\hline (Constante) & 22,73 & 0,83 & \\
\hline Mujer (Referencia: hombre) & $-0,68$ & 0,56 & $-0,02$ \\
\hline Edad en años & $-0,25$ & 0,02 & $-0,26 * * *$ \\
\hline $\operatorname{Edad}^{2}$ & 0,00 & 0,00 & $0,05^{*}$ \\
\hline Tiene hijos <18 años (Referencia: no tiene) & $-1,24$ & 0,69 & $-0,05$ \\
\hline Nivel socioeconómico & 0,03 & 0,00 & $0,49 * * *$ \\
\hline Área rural (Referencia: área urbana) & $-1,47$ & 0,56 & $-0,04 * *$ \\
\hline Capacidad social & 0,15 & 0,02 & $0,18 * * *$ \\
\hline Capacidad política & 0,07 & 0,02 & $0,08 * *$ \\
\hline Capacidad material baja & $-0,81$ & 0,56 & $-0,03$ \\
\hline Capacidad material alta & 1,51 & 0,61 & $0,05^{*}$ \\
\hline (Referencia: capacidad material media) & 22,73 & & \\
\hline
\end{tabular}

Fuente: Encuesta Nacional de Estratificación Social (ENES), 2009.

$\mathrm{R}^{2}=0,46 ; * * * \mathrm{p}<0,001, * * \mathrm{p}<0,01, * \mathrm{p}<0,05$

En el modelo de capacidad cultural se observa que controlando por el resto de las variables, el hecho de pertenecer al género femenino disminuye en promedio la capacidad cultural en 0,7 puntos; sin embargo, este parámetro no es estadísticamente significativo $(\mathrm{p}=0,23)$. En este modelo, las variables explicativas significativamente relacionadas con capacidad cultural de mayor importancia resultan ser el nivel socioeconómico, con una correlación positiva, la edad, que presenta una relación negativa y no lineal, y la capacidad social, con una asociación positiva. Por otra parte, no se encontró evidencia de interacciones relevantes entre mujer y otros predictores.

En el cuadro 7 se aprecia el modelo de capacidad social.
Aquí se observa que controlando por el resto de las variables explicativas, la capacidad social de las mujeres disminuye en poco más de un punto, pero tal como en el caso anterior, este efecto no resulta estadísticamente significativo ( $\mathrm{p}=0,18)$. En este modelo, los efectos más importantes son los de las capacidades cultural y política, tal como sugieren los coeficientes estandarizados. Tampoco se encontró evidencia de interacciones relevantes entre género femenino y otros predictores.

En el cuadro 8 se presenta el modelo para capacidad política.

En este modelo, a diferencia de lo observado en la explicación de las capacidades cultural y social, el género femenino muestra un efecto negativo y estadísticamente

CUADRO 7

Modelo para capacidad social

\begin{tabular}{lccc}
\hline Predictores & $\beta$ & Error típico linealizado & $\beta$ estandarizado \\
\hline (Constante) & 31,28 & 1,06 & $-0,04$ \\
Mujer (Referencia: hombre) & $-1,18$ & 0,88 & $-0,05$ \\
Edad en años & $-0,05$ & 0,04 & $-0,03$ \\
Tiene hijos <18 años (Referencia: no tiene) & $-1,13$ & 0,85 & $0,09 * *$ \\
Nivel socioeconómico & 0,01 & 0,00 & 0,07 \\
Área rural (Referencia: área urbana) & 3,70 & 2,03 & $0,27^{* * *}$ \\
Capacidad cultural & 0,33 & 0,04 & $0,22^{* * *}$ \\
Capacidad política & 0,25 & 0,04 & $-0,10^{* * *}$ \\
Capacidad material baja & $-3,51$ & 1,00 & $-0,07^{* *}$ \\
Capacidad material alta (Referencia: capacidad material media) & $-2,45$ & 0,94 & \\
\hline
\end{tabular}

Fuente: Encuesta Nacional de Estratificación Social (ENES), 2009.

$\mathrm{R}^{2}=0,19 ; * * \mathrm{p}<0,001, * * \mathrm{p}<0,01, * \mathrm{p}<0,05$. 


\begin{tabular}{|c|c|c|c|}
\hline Predictores & $\beta$ & Error típico linealizado & $\beta$ estandarizado \\
\hline (Constante) & 34,98 & 0,95 & \\
\hline Mujer (Referencia: hombre) & $-1,40$ & 0,61 & $-0,05^{*}$ \\
\hline Edad en años & 0,60 & 0,02 & $0,58 * * *$ \\
\hline Edad $^{2}$ & $-0,01$ & 0,00 & $-0,19 * * *$ \\
\hline Tiene hijos < 18 años (Referencia: no tiene) & $-0,01$ & 0,68 & 0,00 \\
\hline Nivel socioeconómico & 0,01 & 0,00 & $0,17 * * *$ \\
\hline Área rural (Referencia: área urbana) & 0,21 & 1,57 & 0,00 \\
\hline Capacidad cultural & 0,10 & 0,04 & $0,09 * *$ \\
\hline Capacidad social & 0,16 & 0,03 & $0,17 * * *$ \\
\hline Capacidad material baja & 0,36 & 0,89 & 0,01 \\
\hline Capacidad material alta & 1,17 & 0,89 & 0,04 \\
\hline (Referencia: capacidad material media) & 34,98 & & \\
\hline
\end{tabular}

Fuente: Encuesta Nacional de Estratificación Social (ENES), 2009.

$\mathrm{R}^{2}=0,38 ; * * * \mathrm{p}<0,001, * * \mathrm{p}<0,01, * \mathrm{p}<0,05$

significativo ( $\mathrm{p}=0,023)$, esto es, en comparación con los hombres, el ser mujer se asocia con una disminución promedio de más de una unidad en el índice de capacidad política. Al comparar los coeficientes estandarizados se observa que en este modelo la edad resulta el predictor de mayor importancia, seguido por el nivel socioeconómico y la capacidad social. Confirmando lo observado anteriormente, no se encontraron interacciones significativas entre sexo y otras variables explicativas.

En resumen, este ejercicio confirma parcialmente las hipótesis del presente trabajo sobre la relación entre género y capacidades. La relación entre ser mujer y una menor capacidad material permanece como un efecto importante incluso luego de controlar por variables sociodemográficas relevantes y otras capacidades. Al repetir este ejercicio con la capacidad cultural y social, la correlación inicialmente observada, si bien mantiene su dirección, tiende a 0 y no resulta estadísticamente significativa. Por otra parte, el efecto negativo de ser mujer en la capacidad política sufre una importante reducción al introducir en el modelo controles tales como edad, nivel socioeconómico, características del hogar y otras capacidades; sin embargo, sigue resultando estadísticamente significativo. Esto implica que se ha encontrado evidencia de un débil efecto neto del sexo en estas capacidades en perjuicio de las mujeres, en comparación con la magnitud de los parámetros significativos de variables como la edad, nivel socioeconómico del hogar o capacidades social y cultural. Por último, al contrario de lo esperado, no se encontró evidencia de interacciones entre sexo y otros predictores de las capacidades, respectivamente, es decir, el funcionamiento de las variables explicativas estudiadas sería estable entre hombre y mujeres.

\section{Discusión y conclusiones}

Los resultados confirman la hipótesis central de este trabajo sobre el menor desarrollo de las capacidades en las mujeres con respecto a los hombres para el caso de la capacidad material y política, mientras que la relación entre género y capacidades cultural y social, pese a funcionar en la dirección esperada, no es suficientemente fuerte. Estas capacidades fueron medidas de acuerdo con los índices elaborados especialmente a partir de las variables de la Encuesta Nacional de Estratificación Social (ENES) de 2009, aplicadas en el marco del proyecto Desigualdades. En cada uno de los índices se combinó una gran cantidad de información a nivel individual —incluidas condiciones, prácticas o conductas y actitudes-, lo que permitió tener una mirada más compleja de cómo se expresan las diferencias entre mujeres y hombres y, a la vez, reducir la dimensionalidad del problema construyendo modelos de mayor parsimonia.

Las mujeres muestran una capacidad material significativamente menor que los hombres, lo que de acuerdo con la definición entraña una menor habilidad de proveerse recursos en forma autónoma y abordar situaciones de riesgo y vulnerabilidad material originadas por el envejecimiento y problemas de salud a través de sistemas de previsión social. La relación entre género femenino y capacidad material se muestra importante incluso al controlar variables independientes relevantes 
y la influencia de las otras capacidades. Estos resultados, que muestran una mayor presencia de las mujeres en las categoría material baja y media, ponen en evidencia el papel estructurante de las relaciones de género de la división sexual del trabajo, que adscribe a las mujeres la responsabilidad casi exclusiva del trabajo doméstico y cuidado no remunerado y al hombre la responsabilidad de proveedor. Esto coloca a las mujeres en una situación de dependencia de los ingresos y probablemente de las elecciones de los otros.

En los modelos explicativos para capacidad social, cultural y política, al controlar por un conjunto de variables explicativas, la desventaja femenina persiste aunque excepcionalmente de manera significativa, contrastando notoriamente con la ostensible menor capacidad material femenina. Esto tiene posibles explicaciones: la mediación de la capacidad material hace referencia a la potencialidad de autonomía de las mujeres y hombres, independientemente de los funcionamientos logrados, a diferencia de lo que sucede en el resto de las capacidades, donde resultó más difícil diferenciar las capacidades de los funcionamientos. Este hallazgo tiene también sentido desde el punto de vista de la teoría de género, que postula que la separación de los espacios privados y públicos y la división sexual del trabajo constituyen la base estructural del orden de género, más estable y resistente a las transformaciones a través del tiempo, como las observadas en el mayor acceso de las mujeres a la educación y a los espacios públicos.

La débil correlación observada entre el género femenino y la capacidad cultural es coherente con los resultados de los estudios de la CEPAL, el Centro de Estudios de la Mujer y el Instituto Nacional de Estadísticas (INE), que coinciden en señalar que en el campo de la educación las brechas por sexo han disminuido más significativamente en los últimos 20 años. En efecto, las mujeres que ingresan al mercado de trabajo tienen mayores niveles de educación que los hombres.

Las mediciones de la capacidad social —recursos sociales que puede movilizar una persona para incrementar su bienestar o enfrentar eventualidades negativas- denotan una mínima desventaja de las mujeres en relación con los hombres. Tal resultado podría deberse a que la encuesta abarca las formas de convivencia social de las zonas rural y urbana: en la primera, las redes comunitarias son más estables, en tanto que en la segunda, el último Informe sobre desarrollo humano en Chile dedicado a los temas de género (PNUD, 2010) mostró que las mujeres han aumentado sus redes sociales a lo largo de los últimos 20 años, debido a los procesos de individuación vinculados a la modernización del país.

Es interesante que la capacidad política sea la otra capacidad donde la desventaja de las mujeres deviene significativa. Así como la división sexual del trabajo y la separación de los ámbitos productivos explican la menor capacidad material de las mujeres, las representaciones culturales sobre hombres y mujeres respecto de campos y capacidades de acción, así como la coexistencia de un doble poder — político y familiar- sobre las mujeres, ponen en evidencia las raíces culturales en que se asienta tal diferencia. Las representaciones adscriben a los hombres a los espacios de la política y las decisiones de país, y a las mujeres en ámbitos de carácter más social o familiar. Estas representaciones son interiorizadas moldeando las motivaciones y expectativas de hombres y mujeres en el campo de la política. Por otra parte y desde una perspectiva institucional, en diversos estudios se han puesto en evidencia los mecanismos de disuasión de la presencia de mujeres en la política. En suma, el conjunto de los resultados muestra cómo el desarrollo de las capacidades es producto de complejos procesos sistémicos relativos a la posición de mujeres y hombres dentro de las relaciones sociales que configuran el orden de género. Algunas de las posiciones ocupadas por las mujeres y los hombres son renuentes al cambio, pues constituyen las bases estructurales del orden de género; otras están más sujetas al efecto de distintas variables y a los procesos de transformación social en curso, tales como la modernización y los procesos de individuación vinculados a ella. 


\section{Bibliografía}

Acosta, E., M. Perticará y C. Ramos (2005), Oferta laboral femenina y cuidado infantil, Washington, D.C., Banco Interamericano de Desarrollo (BID).

Alkire, S. (2002), "Dimensions of human development", World Development, vol. 30, $\mathrm{N}^{\mathrm{o}}$ 2, Amsterdam, Elsevier.

Altman, D. (2004), "Redibujando el mapa electoral chileno: incidencia de factores socioeconómicos y género en las urnas", Revista de ciencia política, vol. 24, $\mathrm{N}^{\circ} 2$, Santiago de Chile, Pontificia Universidad Católica de Chile.

Anand, P., G. Hunter y R. Smith (2005), "Capabilities and well-being: Evidence based on the Sen-Nussbaum approach to welfare", Social Indicators Research, vol. 74, No 1, Nueva York, Springer.

Astelarra, J. (2003), ¿Libres e iguales? Sociedad y política desde el feminismo, Santiago de Chile, Centro de Estudios de la Mujer (CEM).

Berstein, S. y A. Tokman (2005), "Brechas de ingreso entre hombres y mujeres: ¿Perpetuadas o exacerbadas en la vejez?”, Documentos de trabajo, $\mathrm{N}^{\circ} 334$, Santiago de Chile, Banco Central de Chile.

Bertranou, F. (2005), "Pensions and gender in Latin American social protection systems: Where do we stand in the Southern Cone?", documento preparado para la ISSA Expert Group Meeting on Gender, Retirement, and Active Aging: Implications for Social Security in Long-life Societies, Ginebra, Oficina Internacional del Trabajo, junio.

Bertranou, F. y A. Arenas (eds.) (2003), Protección social, pensiones y género, Santiago de Chile, Organización Internacional del Trabajo (OIT).

Bihr, A. y R. Pfefferkorn, (2008), Le système des inégalités, Paris, La Découverte.

Billiet, J.B. (1995), "Church involvement, ethnocentrism, and voting for a radical right-wing party: Diverging behavioral outcomes of equal attitudinal dispositions", Sociology of Religion, vol. 53, $\mathrm{N}^{\circ} 3$, Nueva York, Oxford University Press.

Burchardt, T. y A. Zaidi (2003), "Comparing incomes when needs differ: Equivalisation for the extra costs of disability in the UK", LSE Case Paper, $\mathrm{N}^{\circ}$ 64, Londres, London School of Economics.

Clark, D.A. (2005), "The capability approach: Its development, critiques and recent advances", Working Paper, $\mathrm{N}^{\circ} 32$, Manchester, Global Poverty Research Group.

Clark, D.A. y M. Qizilbash (2005), "Core poverty, basic capabilities and vagueness: An application to the South African context", Working Paper, $\mathrm{N}^{\circ}$ 26, Manchester, Global Poverty Research Group.

Contreras, M.E. (2004), Educación y género. Un desafío a la organización magisterial, Santiago de Chile, Colegio de Profesores de Chile/ Servicio Nacional de la Mujer (SERNAM).

Contreras, D. y G. Plaza (2010), "Cultural factors in women's labor force participation in Chile", Feminist Economics, vol. 16, № 2 , Nueva York, Routledge.

Contreras, D., E. Puentes y D. Bravo (2005), "Female labour force participation in greater Santiago, Chile: 1957-1997. A synthetic cohort analysis", Journal of International Development, vol. 17, $\mathrm{N}^{\circ}$ 2, Mansfield, Universidad de Connecticut.

Dussaillant, F. (2009), “¿Más salas cunas o permisos post natales más largos? Una comparación de alternativas de política para apoyar a la maternidad y a la primera infancia", Documento de trabajo, $\mathrm{N}^{\circ} 377$, Santiago de Chile, Centro de Estudios Públicos (CEP), mayo.

Fraser, N. (1990), “Qué tiene de crítica la teoría crítica? Habermas y la cuestión del género", Teoría feminista y teoría crítica. Ensayos sobre la política de género en las sociedades de capitalismo tardío, S. Benhabib y D. Cornell (eds.), Valencia, Ediciones Alfons El Magnànim.

(1989), Unruly Practices: Power, Discourse, and Gender in Contemporary Social Theory, Minneapolis, Minnesota, University of Minnesota Press.
Fraser, N. y otros (1994), Feminist Contentions: A Philosophical Exchange, Londres, Routledge.

Gamero Requena, J. (2006), "Políticas sociales post-keynesianas. Después del ajuste: ¿qué modelo?”, Confrontaciones monetarias: marxistas y post-keynesianos en América Latina, A. Girón, Buenos Aires, Consejo Latinoamericano de Ciencias Sociales (CLACSO).

Garson, D. (2011), "Binary and Multinomial Logistic Regression" [en línea] http://faculty.chass.ncsu.edu/garson/PA765/logistic.htm

Goldthorpe, J. (1983), "Women and class analysis: In defence of the conventional view", Sociology, vol. 17, No 4, Thousand Oaks, Sage.

Granovetter, M. (1995), Getting a Job: A Study of Contacts and Careers, Chicago, University of Chicago Press.

(1973), "The strength of weak ties", American Journal of Sociology, vol. 78, № 6, Chicago, University of Chicago Press.

Guerrero, E., P. Provoste y A. Valdés (2006a), "Acceso a la educación y socialización de género en un contexto de reformas educativas", Equidad de género y reformas educativas. Argentina, Chile, Colombia, Perú, Patricia Provoste (ed.), Santiago de Chile, Hexagrama Consultoras.

(2006b), "La desigualdad olvidada: Género y educación en Chile", Equidad de género y reformas educativas. Argentina, Chile, Colombia, Perú, Patricia Provoste (ed.), Santiago de Chile, Hexagrama Consultoras.

Guzmán, V. y A. Mauro (2004), "Las trayectorias laborales de mujeres de tres generaciones: Coacción y autonomía", El trabajo se transforma. Relaciones de producción y relaciones de género, R. Todaro y S. Yáñez (eds.), Santiago de Chile, Andros.

Kuklys, W. (2005), Amartya Sen's Capability Approach: Theoretical Insights and Empirical Applications (Studies in Choice and Welfare), Berlin, Springer-Verlag.

Kymlicka, W. (1991), Contemporary Political Philosophy. An Introduction, Nueva York, Oxford University Press.

Larrañaga, O. (2001), "Distribución de ingresos en Chile: 19582001", Documento de trabajo, No 178, Santiago de Chile, Departamento de Economía, Facultad de Ciencias Económicas y Administrativas, Universidad de Chile.

Layte, R., B. Nolan y C. Whelan (2000), "Targeting poverty: Lessons from monitoring Ireland's national anti-poverty strategy", Journal of Social Policy, vol. 29, No 4, Cambridge, Cambridge University Press.

Le Foulon, C. y H. Beyer (2002), "Un recorrido por las desigualdades salariales en Chile", Estudios públicos, vol. 85, Santiago de Chile, Centro de Estudios Públicos (CEP).

Leiva, S. (2000), "El trabajo a tiempo parcial en Chile. ¿Constituye empleo precario? Reflexiones desde la perspectiva de género", serie Mujer y desarrollo, $\mathrm{N}^{\circ} 26$ (LC/L.1301-P), Santiago de Chile, Comisión Económica para América Latina y el Caribe (CEPAL). Publicación de las Naciones Unidas, $\mathrm{N}^{\circ}$ de venta: S.00.II.G.09.

Lombardo, E. (2008), "Gender inequality in politics", International Feminist Journal of Politics, vol. 10, No 1, Nueva York, Routledge.

Majumdar, M. y S. Subramanian (2001), "Capability failure and group disparities: Some evidence from India for the 1980s", Journal of Development Studies, vol. 37, № 5, Nueva York, Routledge.

Martinetti, E. (2000), "A multidimensional assessment of well-being based on Sen's functioning approach", Rivista internazionale di scienze sociali, vol. 108, № 2, Roma, Università Cattolica del Sacro Cuore.

MINEDUC (Ministerio de Educación) (2007), "Indicadores de la educación en Chile 2007", documento preliminar, Santiago de Chile.

(s/f), "Indicadores de la educación en Chile 2006", Santiago de Chile.

Nussbaum, M. (2005), "Well-being, contracts and capabilities", Rethinking Well-Being, L. Manderson (ed.), Perth, API Network. (2003), "Capabilities as fundamental entitlement: Sen and social justice", Feminist Economics, vol. 9, No 2-3, Nueva York, Routledge. 
(2000), Women and Human Development: the Capabilities Approach, Cambridge, Cambridge University Press.

(1995), "Human capabilities, female human beings", Women, Culture and Development, M. Nussbaum y J. Glover (eds.), Oxford, Clarendon Press.

Osborne, R. (2005), "Desigualdad y relaciones de género en las organizaciones: diferencias numéricas, acción positiva y paridad", Política y sociedad, vol. 42, № 2, Madrid, Universidad Complutense de Madrid.

Perticará, M. y I. Bueno (2009), "Brechas salariales por género en Chile: un nuevo enfoque", Revista de la CASEN, No 99 (LC/G.2418-P), Santiago de Chile.

PNUD (Programa de las Naciones Unidas para el Desarrollo) (2010), Género: Los desafíos de la igualdad, Santiago de Chile.

Porras, J.I. y V. Espinoza (2005), "Redes. Enfoques y aplicaciones del análisis de redes sociales (ARS), Santiago de Chile, Universidad Bolivariana.

Qizilbash, M. (1996), "Capabilities, wellbeing and human development”, Journal of Development Studies, vol. 36, Nueva York, Routledge.

Rawls, J. (1971), A Theory of Justice, Cambridge, Harvard University Press.

Ríos, M. (2008), Mujer y política. El impacto de las cuotas de género en América Latina, Santiago de Chile, Catalonia.

Robeyns, I. (2006), "Measuring gender inequality in functionings and capabilities. Findings from the British household panel survey", Gender Disparity: Manifestations, Causes and
Implications, P. Bharati y M. Pal (comps.), Nueva Delhi, Anmol Publishers.

(2003), "Sen's capability approach and gender inequality: Selecting relevant capabilities", Feminist Economics, vol. 9 , $\mathrm{N}^{\circ} 2-3$, Nueva York, Routledge.

Schokkaert, E. y L. Van Ootegem (1990), "Sen's concept of the living standard applied to the Belgium unemployed", Recherches économiques de Louvain, vol. 56, Lovaina, Universidad Católica de Lovaina.

Sen, A. (2000), Desarrollo y libertad, Barcelona, Planeta.

(1985), Commodities and Capabilities, Amsterdam, NorthHolland Press.

(1979), "Personal utilities and public judgments: Or what's wrong with welfare economics?", Economic Journal, vol. 89, Hoboken, Wiley-Blackwell.

(1977), "Social choice theory: A re-examination", Econometrica, vol. 45, No 1, Nueva York, The Econometric Society.

Todaro, R. y S. Yáñez (2004), El trabajo se transforma. Relaciones de producción y relaciones de género, Santiago de Chile, Centro de Estudios de la Mujer (CEM).

Valcárcel, A. (1991), Sexo y filosofía: sobre mujer y poder, Madrid, Anthropos Editorial.

Valdés, T. (2000), De lo social a lo político. La acción de las mujeres latinoamericanas, Santiago de Chile, Lom Ediciones.

Van Hees, M. (2004), "Freedom of choice and diversity of options: Some difficulties", Social Choice and Welfare, vol. 22, $\mathrm{N}^{\circ} 1$, Amsterdam, Elsevier. 